\title{
La eficacia de la biorretroalimentación como tratamiento para el asma. Una revisión sistemática'.
}

\author{
Efficacy of biofeedback as a treatment for asthma. \\ A systematic review
}

\author{
Annielee Pinnock-Branford ${ }^{2}$ \\ Paula Leandro-Argüello ${ }^{3}$ \\ Marijose Sánchez-Burgos ${ }^{4}$ \\ Thannia Fernández-Castro ${ }^{5}$ \\ José Manuel Mora-Benambourg ${ }^{6}$
}

\begin{abstract}
RESUMEN
El asma es una enfermedad del sistema respiratorio cuyos síntomas incluyen dificultad para respirar, opresión del pecho, tos y sibilancias. Distintos tratamientos han sido investigados y probados para su control, siendo el tratamiento con esteroides antiinflamatorios el más utilizado. No obstante, la biorretroalimentación se ha posicionado como un tratamiento con relevancia y efectividad clínica. Este estudio realizó una revisión sistemática que contó con 16 artículos sobre el uso de la biorretroalimentación como tratamiento para el asma. Los criterios de elegibilidad fueron: a) La variable dependiente debía ser asma y utilizar biorretroalimentación como tratamiento. b) Ser un estudio experimental o cuasi experimental y brindar el tamaño de la muestra. c) Tener grupo control, pre y postest. Los resultados sugieren que el uso de biorretroalimentación de respiración y variabilidad de la tasa cardiaca son efectivos cuando se trata de asma leve a moderada y, aún más, si se acompaña de medicación y otros tratamientos como relajación. Esto implica que podría ser considerada como tratamiento probablemente eficaz en casos donde el tratamiento farmacológico no sea viable. La mayor limitación encontrada fue la falta de estudios que contaran con los criterios de calidad necesarios para un meta análisis, por lo que se optó realizar una revisión sistemática.
\end{abstract}

Palabras clave: Revisión sistemática, Asma, biofeedback.

\footnotetext{
1 Trabajo realizado para el curso PS- 1049 Módulo de Psicofisiología Aplicada y biofeedback en los Trastornos de Ansiedad de la Escuela de Psicología de la Universidad de Costa Rica.

${ }^{2}$ Universidad de Costa Rica. Facultad de Ciencias Sociales. Bachiller en Psicología. San José, Costa Rica. Correo electrónico: anniebranford@ gmail.com ORCID: https://orcid.org/0000-0002-9367-1123

${ }^{3}$ Universidad de Costa Rica. Facultad de Ciencias Sociales. Bachiller en Psicología. San José, Costa

Rica. Correo electrónico: pauleandro21 @ gmail.com. ORCID: https://orcid.org/0000-0001-7564-5565

${ }^{4}$ Universidad de Costa Rica. Facultad de Ciencias Sociales. Bachiller en Psicología. San José, Costa

Rica. Correo electrónico: marijosesanchez97@ gmail.com ORCID: https://orcid.org/0000-0001-88527293

${ }^{5}$ Universidad de Costa Rica. Facultad de Ciencias Sociales. Bachiller en Psicología. San José, Costa Rica. Correo electrónico: thannia161294@ gmail.com ORCID: https://orcid.org/0000-0002-3159-4361

${ }^{6}$ Universidad de Costa Rica. Facultad de Ciencias Sociales. Licenciado en Psicología. San José, Costa Rica. Correo electrónico: jose.mora_bena@ucr.ac.cr ORCID: https://orcid.org/0000-0002-5557-9385 Doi: $10.15517 /$ WL.V1611.45971

Recepción: 9/5/2020 Aceptación: 15/1/2020
} 


\begin{abstract}
Asthma is a disease of the respiratory system whose symptoms include shortness of breath, chest tightness, coughing, and wheezing. Different treatments have been investigated and tested to control it, the treatment with anti-inflammatory steroids being the most used. However, biofeedback has been positioned as a treatment with clinical relevance and effectiveness. This study conducted a systematic review that included 16 articles on the use of biofeedback as a treatment for asthma. The eligibility criteria followed were: a) The dependent variable had to be asthma and use biofeedback as treatment. b) Be an experimental or quasiexperimental study and provide the sample size. c) Have a control group, pre and post-test. The results suggest that the use of respiratory biofeedback and heart rate variability is effective when it comes to mild and moderate asthma and, even more so, when accompanied by medication and other treatments such as relaxation. This implies that it could be considered as a probably effective treatment in cases where pharmaceutical treatment is not a viable option. The biggest limitation found was the lack of studies that had the necessary quality criteria for a meta-analysis, which is why a systematic review was chosen.
\end{abstract}

Key Words: Sistematic review, Asthma, Biofeedback.

\title{
Introducción
}

El asma es una enfermedad del sistema respiratorio que se caracteriza por la hinchazón y, por lo tanto, estrechez de las vías respiratorias, lo que causa síntomas como dificultad para respirar, opresión en el pecho, tos y sibilancias (Lehrer, 2012). Está condición puede afectar a personas de todas las edades, aunque suele ser más frecuente en niños y niñas en edades escolares. De acuerdo con Jiménez (2019), la Organización Mundial de la Salud estima que alrededor de 339 millones de personas la padecen y el Reporte Global de Asma indica que, para el 2018, solo en Costa Rica 42.606 niños y niñas entre los seis y catorce años sufren de esta, posicionando al país por encima del promedio de América Latina.

Actualmente el tratamiento más utilizado para el asma es el uso de agentes antiinflamatorios, como la inhalación de esteroides, medicamentos que aparecen en la vasta mayoría de protocolos y guías para tratar el asma persistente (Kern-Buell et al. 2000, Lehrer 2012, Lehrer et al. 2004). No obstante, el uso prolongado de esteroides no solo implica un gasto significativo para las personas que los consumen, sino que, además, puede traer efectos adversos sobre la salud de los y las pacientes, como la candidiasis oral, glaucoma y/o disminución de la densidad ósea. Asimismo, puede generar efectos no deseados que dificultan la adherencia al tratamiento, como tos, formación de moretones con facilidad, disfonía, entre otros (Lehrer et al. 2004). Debido a lo anterior, a partir de la década de los 70 se han explorado diferentes formas de tratamiento, especialmente para 
aquellos casos de mayor severidad, buscando encontrar un equilibrio entre el uso prolongado de medicamentos y una baja en la severidad de los síntomas asmáticos.

Dentro de estos tratamientos, el uso de la biorretroalimentación ha mostrado ser una alternativa prometedora con evidencia que le apoya (Kern-Buell et al. 2000, Lehrer 2012, Lehrer et al. 2004, Ritz et al. 2004). Lo anterior debido a que, como lo explica Lehrer (2012), puede ser una opción que altere efectivamente los parámetros de funcionamiento de las vías respiratorias y, a su vez, un tratamiento menos prolongado en el tiempo que los y las pacientes podrían preferir en lugar del tratamiento farmacológico estándar. Se han probado diversas modalidades de biorretroalimentación para esta condición, desde el uso de electromiografía para la medición de la tensión de los músculos faciales, protocolos de neurofeedback o protocolos de resistencia respiratoria con el método de oscilación forzada. Sin embargo, es importante tomar en consideración que la literatura pareciera ser consistente en el hecho de que el entrenamiento en biorretroalimentación para aumentar la variabilidad de la tasa cardiaca (HRV-BF, por sus siglas en inglés) es el que ha mostrado mayor eficacia frente a otras modalidades, evidenciando de manera consistente mayores efectos clínicamente significativos y positivos (Kondo et al. 2019, Lehrer 2012, Nelson et al. 2020).

No obstante, si bien es cierto la biorretroalimentación se ha posicionado como un tratamiento eficaz para distintas condiciones (Kondo et al. 2019), aún existe un vacío de información referente a este tratamiento para el asma. La mayoría de los estudios encontrados datan desde la década de los 70s y 80s, lo que se puede estar reflejando en la falta de revisiones sistemáticas o metaanálisis que brinden un panorama general del tema en la actualidad. Por esta razón, el objetivo de esta revisión sistemática es determinar la eficacia del uso de biorretroalimentación, en sus diversas modalidades, como tratamiento para el asma. 


\section{Metodología}

\section{Fuentes de información}

La búsqueda y revisión de material bibliográfico se llevó a cabo desde el 29 de abril del 2020 hasta el 27 de mayo del 2020. Se realizó en las bases de datos EbscoHost, Google Scholar, Springerlink, Elsevier, Science Direct, PsycNet, ProQuest y Pubmed; así como en los sitios web de Journal of Asthma, National Center of Biotechnology Information y Journal of Applied Psychophysiology and Biofeedback. Se utilizaron las siguientes palabras clave: "Biofeedback AND asthma", "HRV AND Asthma", "Biofeedback AND Trial AND Asthma" y "Biofeedback AND Asthma AND Treatment". Adicionalmente, se revisaron las referencias de los artículos encontrados con el propósito de expandir la búsqueda y revisión bibliográfica. Se creó una plantilla en Excel donde se registró la fecha de búsqueda, la base de datos o sitio web revisado y las palabras clave utilizadas.

\section{Criterios de elegibilidad}

Para la revisión sistemática de literatura se establecieron los siguientes criterios de elegibilidad: a) La variable dependiente debía ser asma y utilizar biorretroalimentación como tratamiento. b) Ser un estudio experimental o cuasi experimental y brindar el tamaño de la muestra. c) Tener un grupo control, pre y postest. d) En inglés o en español. En un principio se consideró el año de publicación como un criterio de elegibilidad (del 2010 en adelante), pero este criterio tuvo que ser omitido debido a la falta de material bibliográfico que cumpliera con los criterios de elegibilidad previamente mencionados. De esta forma, se revisó material publicado desde el año 1973 hasta el 2020. La Figura 1 resume el proceso de selección de los estudios. 


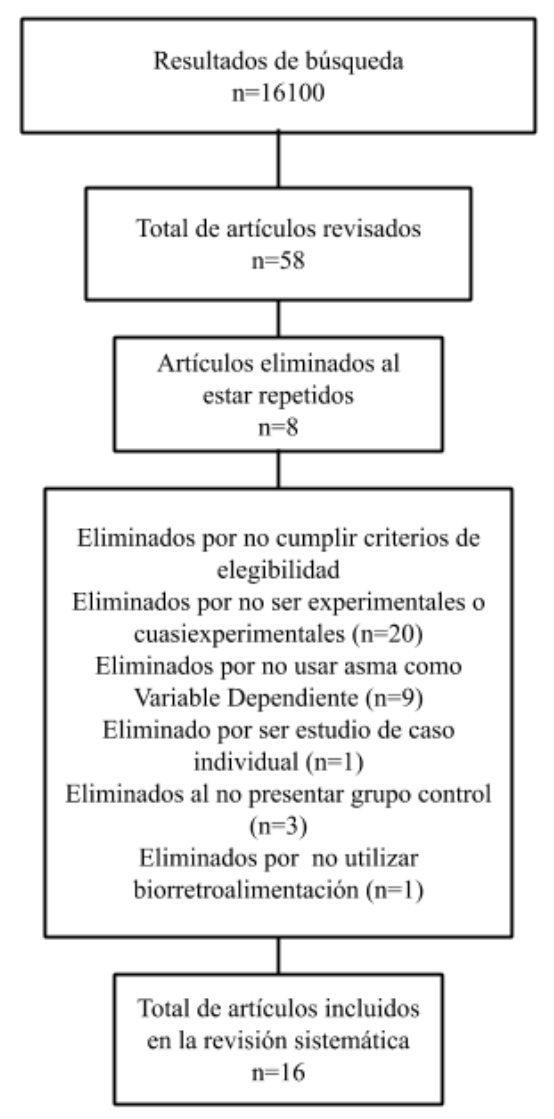

Figura 1. Proceso de selección de estudios

\section{Extracción de datos}

Para la codificación de los estudios se anotó el autor, año de publicación, tipo de estudio, variable dependiente, tamaño de muestra, tamaño de los grupos; así como si los estudios seleccionados utilizaban asignación al azar, grupo control y mediciones pre y postest. Por último, se anotaron las distintas variables moderadoras, las cuales se detallan a continuación: medicación, severidad del asma, tipo de biorretroalimentación, uso de otro tratamiento, número y duración de sesiones, edad, estado de ánimo y calidad del estudio. La Tabla 1 resume la estrategia de codificación empleada. 
Tabla 1. Estrategia de codificación

\begin{tabular}{|c|c|c|}
\hline Variable & $\begin{array}{l}\text { Escala de } \\
\text { medición }\end{array}$ & Estrategia de codificación \\
\hline Autor & NA & Referencia \\
\hline Año & Continua & Año de publicación \\
\hline Tipo de estudio & Categórica & $\begin{array}{c}\text { Experimental } \\
\text { Cuasi Experimental }\end{array}$ \\
\hline Variable dependiente & Categórica & $\begin{array}{c}\text { Asma } \\
\text { No asma }\end{array}$ \\
\hline Tamaño de la muestra & Continua & Número total de participantes \\
\hline Tamaño de los grupos & Continua & $\begin{array}{c}\text { Número de personas por grupo } \\
\text { experimental y control }\end{array}$ \\
\hline Medicación & Categórica & $\begin{array}{l}\text { Sí recibe } \\
\text { No recibe }\end{array}$ \\
\hline Uso de Medicación & Categórica & $\begin{array}{l}\text { Parte del estudio } \\
\text { Control personal }\end{array}$ \\
\hline Tipo de medicación & Categórica & Tipo de medicación \\
\hline Severidad del asma & Categórica & $\begin{array}{l}\text { No severa } \\
\text { Leve } \\
\text { Moderada } \\
\text { Severa }\end{array}$ \\
\hline
\end{tabular}


Wimblu, Rev. Estud. de Psicología UCR, 16(1) 2021 (Enero-Junio): 29-50 /ISSN: 1659-2107

Tipo de

Continua

Tipo de biorretroalimentación utilizada

biorretroalimentación

Otro tratamiento

Continua

Tipo de tratamiento adicional a la

biorretroalimentación

Edad

Continua

Media de edad de las personas

participantes

Número de sesiones

Continua

Número de sesiones en el grupo

experimental

Duración de las sesiones Continua

Tiempo de cada sesión

Duración total del

Continua

Total de horas sumadas

entrenamiento

Estado de ánimo

Categórica

Sí lo toma en cuenta

No lo toma en cuenta

Calidad

Continua

Puntaje total de calidad

Fuente: Elaboración propia.

\section{Evaluación de la calidad}

Para la evaluación de la calidad se utilizó una adaptación de la escala propuesta por Luna, Kruchten, Pedrosa, Neto y de Moura (2014). Cada estudio fue evaluado en una escala de 0 a 24 puntos, según si cumplían explícitamente con el criterio ( 2 puntos), implícitamente (1 punto) o no lo cumplían del todo (0 puntos).

\section{Resultados}

Como se especificó en las secciones anteriores, en la búsqueda original se encontraron alrededor de 16000 estudios acerca del uso de la biorretroalimentación como 
tratamiento para el asma. Sin embargo, luego de una revisión exhaustiva y verificando que los estudios encontrados cumplieran con los criterios de elegibilidad propuestos, se realizó el estudio en cuestión con 16 de ellos. A continuación, se exponen los resultados encontrados en el proceso de revisión sistemática a partir de los criterios especificados en apartados anteriores.

En términos generales, los 16 artículos que se utilizaron para la revisión sistemática se ubicaron en un rango entre 16 y 22 en la evaluación de calidad $(M=19.81$, $D E=1.83)$. La Tabla 2 muestra los resultados de la evaluación de la calidad para cada estudio.

Tabla 2. Resultados de la evaluación de la calidad por estudio

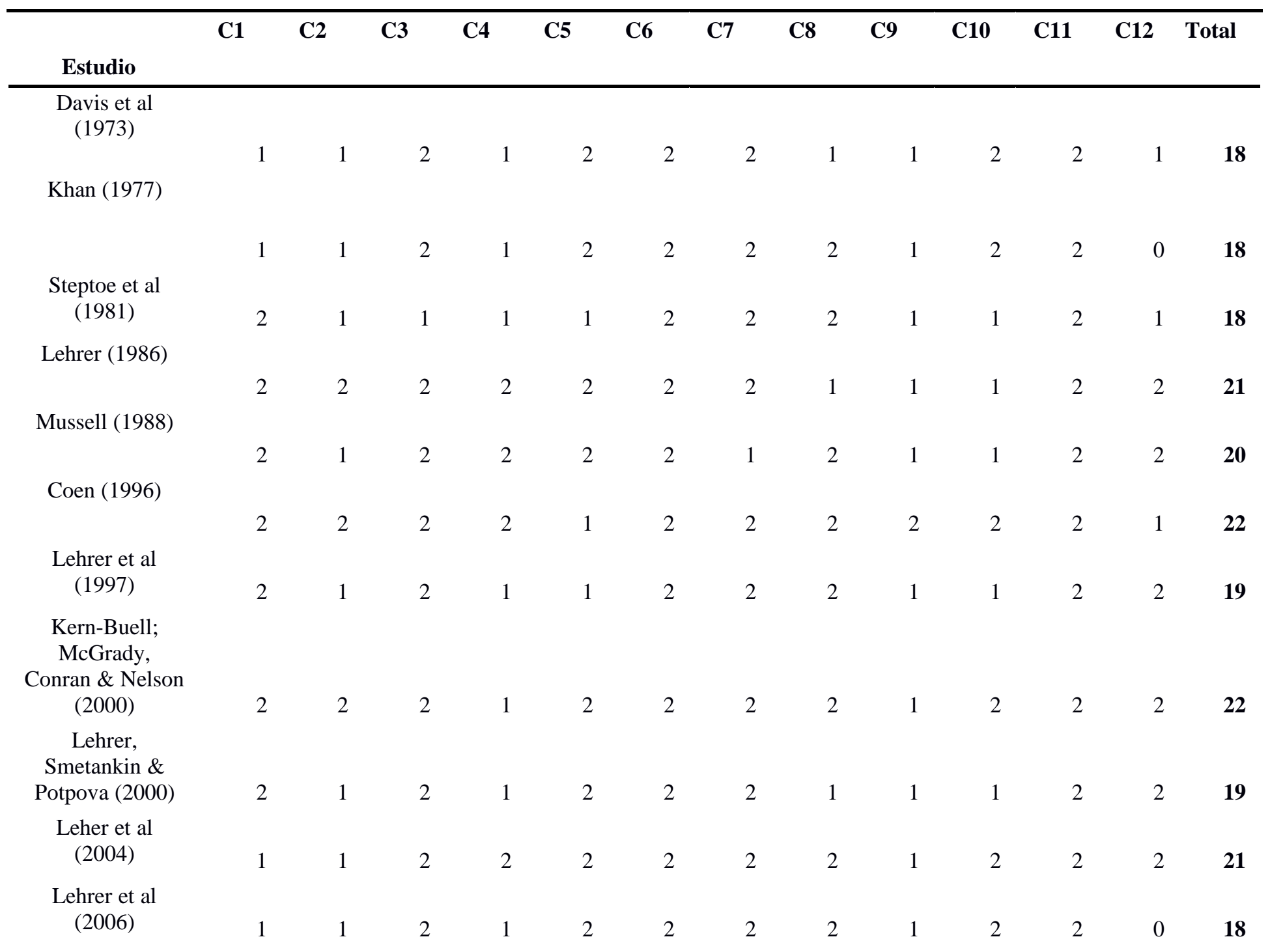




\section{Dremum}

Wimblu, Rev. Estud. de Psicología UCR, 16(1) 2021 (Enero-Junio): 29-50 /ISSN: 1659-2107

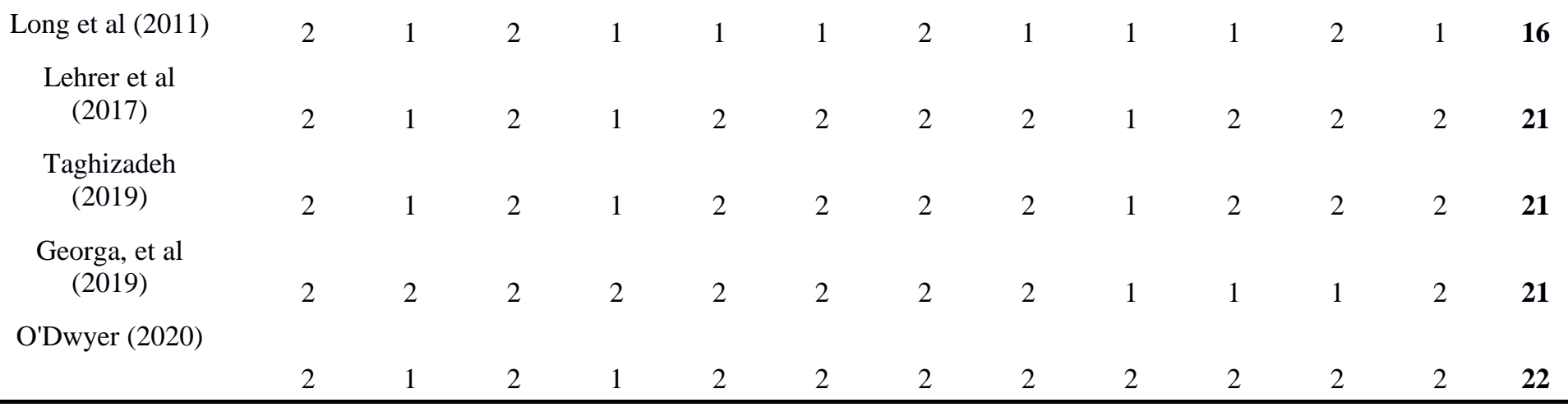

Nota: [C1. Is there a theoretical background about the topics of the study?], [C2. Is there a clear definition of the justifications of the study?], [C3. Is there a clear definition of the study objectives?], [C4. Is there a clear definition of the research question (RQ) and/or the hypothesis of the study?], [C5. Is there an adequate description of the context in which the research was carried out?], [C6. Is there an adequate description of the sample used and the methods for identifying and recruiting the sample?], [C7. Are used and described appropriate data collection methods?], [C8. Is there an adequate description of the methods used to analyze data and appropriate methods for ensuring the data analysis were grounded in the data?], [C9. Is provided by the study clearly answer or justification about RQ / hypothesis?], [C10. Is provided by the study clearly stated findings with credible results?], [C11. Is provided by the study justified conclusions?], [C12. Is provided by the study discussion about validity threats?]

Según la evaluación de calidad realizada para cada estudio, se constata que, en su mayoría, existen antecedentes teóricos claros para los objetivos definidos en cada estudio. Con respecto a la metodología, la mayoría explica apropiadamente el contexto del estudio, la muestra y los criterios de inclusión de la misma. En esta misma línea se seleccionaron métodos apropiados para la recolección y análisis de datos, lo que conlleva a que la mayoría de estudios presenten resultados creíbles y conclusiones justificadas. En general, se aprecia que los estudios son rigurosos y sólo se cumple de manera implícita en unos pocos la definición y justificación de la hipótesis, al igual que en pocos casos se omite la discusión sobre las amenazas a la validez.

No obstante, tomando en consideración que dentro de los criterios de elegibilidad el año de publicación de los artículos se amplió y se incluyeron estudios realizados en la década de los 70 y 80, siendo pioneros en la investigación sobre la biorretroalimentación como tratamiento para el asma, es importante resaltar la evolución de los mismos a través de los años. De esta forma, es posible observar que la principal diferencia se encuentra en la calidad metodológica de los estudios. Aquellos estudios realizados entre finales del siglo pasado e inicios del presente, son menos rigurosos en cuanto a los procedimientos y protocolos utilizados en la investigación. Así, en comparación con estudios realizados en la última década, aquellos más antiguos presentan pocos detalles en cuanto a 
características de la muestra reclutada, criterios de selección y el o los protocolos utilizados para el tratamiento, lo que es posible constatar en las puntuaciones obtenidas en la evaluación de calidad, donde los artículos más antiguos en promedio obtuvieron una puntuación más baja.

Por otra parte, con relación a las personas participantes, las muestras variaron en términos de grupo etario, pues se encontraban dentro del rango de 15 a 94 años y estaban compuestas por hombres y mujeres $(\mathrm{N}=553)$. De los 16 estudios, 10 fueron conducidos en Estados Unidos y el resto se llevaron a cabo en países como Irlanda, Inglaterra, Grecia e Irán. Cuatro de ellos utilizaron un diseño cuasi-experimental y el resto un diseño experimental. El número de sesiones varió entre 1 y 18 y la duración de las mismas entre 20 minutos a 1 hora 30 minutos, siendo la mayoría de 30 minutos.

Se tomaron en cuenta ciertas variables moderadoras para el análisis. Entre estas, se incluyeron el tipo de biorretroalimentación utilizada en el estudio, así como la severidad del asma y la presencia de otro tratamiento, ya sea farmacológico o de cualquier otra índole. A partir de este análisis, se encontró que los tipos de biofeedback varían desde la electromiografía (EMG) hasta la variabilidad de la tasa cardíaca (HRV, por sus siglas en inglés), incluyendo respiración (RESP), electroencefalografía (EEG), temperatura periférica (TP) y arritmia sinusal respiratoria (ASR). Por otra parte, la severidad del asma se encontraba en un rango entre leve y severa, incluyendo otras tipologías como intermitente, persistente y crónica.

Asimismo, 6 estudios acompañaron la biorretroalimentación con entrenamientos en relajación de diferentes tipos, entre estas la relajación progresiva de Jacobson, con imaginería, respiración profunda y respiración abdominal. Un estudio acompañó la biorretroalimentación con mediciones de electroencefalografía y 10 estudios incluyeron abordaje farmacológico. Entre los fármacos utilizados se encuentran el inhalador, los corticosteroides, esteroides y broncodilatadores. De igual manera, se mencionan medicamentos más específicos como el Albuterol, Fluticasone, Isoprotenol, Intal y Bexatol. Las características de los estudios se resumen en la Tabla 3. 
Tabla 3. Características de los estudios

\begin{tabular}{|c|c|c|c|c|c|c|c|c|}
\hline Autor y Año & País & Muestra & $\begin{array}{l}\text { Grupo } \\
\text { Etario }\end{array}$ & Diseño & $\begin{array}{c}\text { Tipo de } \\
\text { biorretroal } \\
\text { imentación }\end{array}$ & $\begin{array}{l}\text { Severidad } \\
\text { Asma }\end{array}$ & Medicación & $\begin{array}{c}\text { Otro } \\
\text { tratamiento }\end{array}$ \\
\hline Davis et al (1973) & $\begin{array}{l}\text { Inglater } \\
\text { ra }\end{array}$ & 24 & $\begin{array}{c}\text { Niñez a } \\
\text { adolescencia }\end{array}$ & $\begin{array}{l}\text { Cuasi- } \\
\text { experi } \\
\text { mental }\end{array}$ & EMG & $\begin{array}{l}\text { Severa y } \\
\text { no severa }\end{array}$ & $\begin{array}{c}\text { Sí utilizan } \\
\text { (Corticosteroid } \\
\text { es) }\end{array}$ & ------ \\
\hline Khan (1977) & EEUU & 80 & $\begin{array}{c}\text { Niñez a } \\
\text { adolescencia }\end{array}$ & $\begin{array}{l}\text { Experi } \\
\text { mental }\end{array}$ & RESP & $\begin{array}{l}\text { Severa y } \\
\text { no severa }\end{array}$ & $\begin{array}{l}\text { Sí utilizan } \\
\text { (Isoprotenol) }\end{array}$ & ------ \\
\hline $\begin{array}{l}\text { Steptoe et al } \\
(1981)\end{array}$ & $\begin{array}{l}\text { Inglater } \\
\text { ra }\end{array}$ & 24 & $\begin{array}{c}\text { Adultez } \\
\text { emergente } \\
\text { hasta } \\
\text { adultez } \\
\text { media }\end{array}$ & $\begin{array}{l}\text { Experi } \\
\text { mental }\end{array}$ & RESP & ------ & $\begin{array}{l}\text { Sí utilizan } \\
\text { (Bexatol) }\end{array}$ & ------ \\
\hline Lehrer (1986) & EEUU & 22 & $\begin{array}{l}\text { Adultez } \\
\text { media }\end{array}$ & $\begin{array}{l}\text { Cuasi- } \\
\text { experi } \\
\text { mental }\end{array}$ & EMG & $\begin{array}{c}\text { Leve, } \\
\text { severa y } \\
\text { moderada }\end{array}$ & $\begin{array}{c}\text { Sí utilizan } \\
\text { (Broncodilatad } \\
\text { ores } \\
\text { Theophylline y } \\
\text { esteroides } \\
\text { orales) }\end{array}$ & ------ \\
\hline Mussell (1988) & $\begin{array}{l}\text { Inglater } \\
\text { ra }\end{array}$ & 15 & ------- & $\begin{array}{l}\text { Cuasi- } \\
\text { experi } \\
\text { mental }\end{array}$ & Trachea Noise & ------- & $\begin{array}{c}\text { Sí utilizan } \\
\text { (Broncodilatad } \\
\text { or) }\end{array}$ & ------ \\
\hline Coen (1996) & EEUU & 20 & $\begin{array}{l}\text { Adolescenci } \\
\text { a a adultez } \\
\text { media }\end{array}$ & $\begin{array}{l}\text { Experi } \\
\text { mental }\end{array}$ & TEMP & ------- & $\begin{array}{l}\text { Sí utilizan (no } \\
\text { especifican) }\end{array}$ & $\begin{array}{c}\text { Relajación } \\
\text { asistida por BF } \\
\text { y } \\
\text { entrenamiento } \\
\text { en respiración } \\
\text { profunda }\end{array}$ \\
\hline $\begin{array}{l}\text { Lehrer et al } \\
\text { (1997) }\end{array}$ & EEUU & 17 & $\begin{array}{l}\text { Adultez } \\
\text { emergente } \\
\text { hasta } \\
\text { adultez } \\
\text { mayor }\end{array}$ & $\begin{array}{l}\text { Experi } \\
\text { mental }\end{array}$ & EMG y RESP & ------ & $\begin{array}{c}\text { Sí utilizan } \\
\text { (Theophylline } \\
\text { y esteroides } \\
\text { orales) }\end{array}$ & $\begin{array}{l}\text { Relajación } \\
\text { progresiva de } \\
\text { Jacobson }\end{array}$ \\
\hline $\begin{array}{c}\text { Lehrer, } \\
\text { Smetankin \& } \\
\text { Potapova (2000). }\end{array}$ & EEUU & 20 & $\begin{array}{c}\text { Niñez y } \\
\text { adolescencia }\end{array}$ & $\begin{array}{l}\text { Experi } \\
\text { mental }\end{array}$ & ASR & $\begin{array}{l}\text { Leve a } \\
\text { moderada }\end{array}$ & No utilizan & ------ \\
\hline
\end{tabular}


Wimblu, Rev. Estud. de Psicología UCR, 16(1) 2021 (Enero-Junio): 29-50 /ISSN: 1659-2107

\begin{tabular}{|c|c|c|c|c|c|c|c|c|}
\hline $\begin{array}{c}\text { Kern-Buell; } \\
\text { McGrady, } \\
\text { Conran \& Nelson } \\
\text { (2000) }\end{array}$ & EEUU & 16 & $\begin{array}{l}\text { Adolescenci } \\
\text { a y adultez } \\
\text { emergente }\end{array}$ & $\begin{array}{l}\text { Experi } \\
\text { mental }\end{array}$ & EMG & $\begin{array}{l}\text { Leve a } \\
\text { moderada }\end{array}$ & $\begin{array}{l}\text { Sí utilizan (no } \\
\text { especifican) }\end{array}$ & $\begin{array}{l}\text { Entrenamiento } \\
\text { en relajación }\end{array}$ \\
\hline Leher et al (2004) & EEUU & 94 & $\begin{array}{c}\text { Adultez } \\
\text { emergente } \\
\text { hasta } \\
\text { adultez } \\
\text { mayor }\end{array}$ & $\begin{array}{l}\text { Experi } \\
\text { mental }\end{array}$ & $\begin{array}{l}\text { HRV y HRV } \\
\text { con } \\
\text { respiración } \\
\text { abdominal }\end{array}$ & $\begin{array}{c}\text { Media } \\
\text { intermitent } \\
\text { e, media } \\
\text { persistente, } \\
\text { moderada } \\
\text { y severa }\end{array}$ & $\begin{array}{c}\text { Sí utilizan } \\
\text { (Fluticasone) }\end{array}$ & \\
\hline $\begin{array}{l}\text { Lehrer et al } \\
\quad(2006)\end{array}$ & EEUU & 45 & $\begin{array}{l}\text { Adolescenci } \\
\text { a hasta } \\
\text { adultez } \\
\text { mayor }\end{array}$ & $\begin{array}{l}\text { Cuasi- } \\
\text { experi } \\
\text { mental }\end{array}$ & HRV & ------ & $\begin{array}{l}\text { Sí utilizan (no } \\
\text { especifican) }\end{array}$ & $\begin{array}{c}\text { Entrenamiento } \\
\text { en respiración } \\
\text { abdominal }\end{array}$ \\
\hline Long et al (2011) & EEUU & 22 & $\begin{array}{c}\text { Niñez y } \\
\text { adolescencia }\end{array}$ & $\begin{array}{l}\text { Experi } \\
\text { mental }\end{array}$ & $\begin{array}{l}\text { RESP y } \\
\text { TEMP }\end{array}$ & $\begin{array}{l}\text { Moderada } \\
y \\
\text { persistente }\end{array}$ & $\begin{array}{l}\text { Sí utilizan (no } \\
\text { especifican) }\end{array}$ & $\begin{array}{l}\text { Relajación } \\
\text { progresiva de } \\
\text { Jacobson y } \\
\text { relajación con } \\
\text { imaginería }\end{array}$ \\
\hline $\begin{array}{l}\text { Lehrer et al } \\
\quad(2017)\end{array}$ & EEUU & 68 & $\begin{array}{c}\text { Adultez } \\
\text { emergente } \\
\text { hasta } \\
\text { adultez } \\
\text { mayor }\end{array}$ & $\begin{array}{l}\text { Experi } \\
\text { mental }\end{array}$ & HRV y EEG & $\begin{array}{c}\text { Leve a } \\
\text { moderada } \\
\text { persistente }\end{array}$ & $\begin{array}{l}\text { Sí utilizan } \\
\text { (Abulterol y } \\
\text { esteroides } \\
\text { orales) }\end{array}$ & ------- \\
\hline $\begin{array}{l}\text { Taghizadeh } \\
\text { (2019) }\end{array}$ & Irán & 44 & $\begin{array}{c}\text { Adultez } \\
\text { emergente }\end{array}$ & $\begin{array}{l}\text { Experi } \\
\text { mental }\end{array}$ & HRV & Leve & $\begin{array}{c}\text { Sí utilizan } \\
\text { (Agonista de } \\
\text { beta y } \\
\text { corticosteroide } \\
\text { s) }\end{array}$ & Medición ECG \\
\hline $\begin{array}{l}\text { Georga, et al } \\
\text { (2019) }\end{array}$ & Grecia & 42 & $\begin{array}{l}\text { Adultez } \\
\text { emergente } \\
\text { hasta } \\
\text { adultez } \\
\text { mayor }\end{array}$ & $\begin{array}{l}\text { Experi } \\
\text { mental }\end{array}$ & $\begin{array}{c}\text { GSR, RESP y } \\
\text { HRV }\end{array}$ & $\begin{array}{l}\text { Intermitent } \\
\text { e a media } \\
\text { crónica }\end{array}$ & $\begin{array}{l}\text { Sí utilizan (no } \\
\text { especifican) }\end{array}$ & $\begin{array}{l}\text { Relajación } \\
\text { progresiva de } \\
\text { Jacobson }\end{array}$ \\
\hline O'Dwyer (2020) & Irlanda & 152 & $\begin{array}{l}\text { Adultez } \\
\text { emergente } \\
\text { hasta } \\
\text { adultez } \\
\text { mayor }\end{array}$ & $\begin{array}{l}\text { Experi } \\
\text { mental }\end{array}$ & RESP & ------ & $\begin{array}{l}\text { Sí utilizan } \\
\text { (Inhalador) }\end{array}$ & ------ \\
\hline
\end{tabular}

Como se puede observar en la Tabla 3, es notable una evolución en el tipo de biorretroalimentación utilizada, de acuerdo con la evidencia científica obtenida a partir de investigaciones anteriores. Las primeras investigaciones realizadas en la década de los 
70 y hasta los primeros años del siglo XXI, apuntaban al uso de protocolos con EMG y RESP principalmente, y únicamente combinaban la biorretroalimentación con medicación, con lo que rara vez se incluía otro tipo de tratamiento. De igual manera, tenían como grupo etario de estudio mayoritariamente niñez y adolescencia. Para mediados del siglo, partiendo de que las investigaciones anteriores no mostraron evidencia clínicamente significativa, se dio un auge en la investigación utilizando protocolos con HRV o una combinación entre este y otras modalidades de biorretroalimentación u otros tratamientos. Asimismo, la población de estudio pasó a ser mayoritariamente personas en la adultez media.

En cuanto al tipo de biorretroalimentación, se logra constatar a partir de la revisión bibliográfica que la mayoría de protocolos se agrupan en tres diferentes modalidades o metodologías de tratamiento y entrenamiento en biorretroalimentación, siendo EMG, HRV o RESP las más utilizadas. De igual manera, se observa que en la mayoría de investigaciones analizadas, el uso de la biorretroalimentación se da en conjunto con otras variables que son parte del tratamiento, como lo son protocolos de relajación muscular progresiva, entrenamientos en respiración abdominal y el uso de medicamentos, ya sea dados como parte del estudio o bien, que las personas participantes ya consumían y no se restringe su uso dentro de la investigación.

En cuanto a la eficacia de la biorretroalimentación como tratamiento para el asma propiamente, los grupos experimentales en donde este era parte del tratamiento mostraron una disminución significativa en el uso de medicamentos, frecuencia y duración de los ataques del asma, después de terminados los protocolos. Es importante recalcar que en la mayoría de casos, la severidad del asma se ve disminuida de manera significativa si el tratamiento con biorretroalimentación se acompaña de otros tratamientos, como los que se han venido mencionando: entrenamientos en respiración abdominal, relajación muscular progresiva u otros tipos de relajación. En promedio, mejoras de este tipo se comienzan a observar a partir de la tercera o quinta sesión de entrenamiento en biorretroalimentación.

Entrando en la caracterización de otras variables como lo es la severidad del asma, se encontró que los pacientes que obtienen mejores resultados son aquellos que cuentan 
con el diagnóstico de asma no severo, mientras que aquellos con un diagnóstico de asma severo no tienden a mostrar resultados clínicamente significativos. Si bien es cierto la biorretroalimentación muestra ser eficaz a la hora de tratar los síntomas del asma, la presencia de medicamentos juega un papel importante en el manejo de estos y por lo tanto se rescata como una conclusión importante en la mayoría de investigaciones que no se podría descartar el uso de medicamentos como parte del tratamiento.

Por último, factores específicos como la edad no parecen tener una influencia significativa en la mejoría o efectividad del tratamiento. Con respecto al estado de ánimo o precursores emocionales de ataques de asma, en los estudios en los que estos se tomaron como una variable de interés se reportó una disminución en los mismos después del entrenamiento con biorretroalimentación, principalmente en aquellos en los que se utilizan otros tratamientos complementarios como el uso de relajaciones en general.

\section{Discusión}

De manera general, es posible encontrar una amplia variedad de literatura respecto al uso de la biorretroalimentación como tratamiento para el asma. Sin embargo, la mayoría de estas son publicaciones de tipo descriptivo, especialmente aquellas divulgadas en la última década. Por el contrario, son escasas aquellas que se centran en explorar de manera experimental intervenciones prácticas. Esto pareciera mostrar que el auge de investigación en la temática se dió a finales del siglo pasado e inicios del presente. Asimismo, si bien la cantidad de artículos existentes en el tema de asma y su relación con la biorretroalimentación son muchos; al momento de buscar artículos que cumplan criterios de calidad para realizar una revisión sistemática o un metaanálisis, los que cumplen con los criterios necesarios son muy pocos.

A pesar de estas limitaciones, dentro de los principales hallazgos se destaca que, a través de los años, se ha probado que el uso de varios tipos de biorretroalimentación resulta efectivo para tratar los síntomas del asma, tales como la biorretroalimentación con electromiografía, respuesta respiratoria y variabilidad de la tasa cardiaca. Esto coincide 
con los hallazgos de Coen et al. (1996), Kern-Buell et al. (2000) y O’Dwyer et al. (2020), en tanto, según estos autores, la mayoría de los resultados de los entrenamientos demostraron ser efectivos para la reducción de algunos de los síntomas asociados al asma. A su vez, estos autores destacan que no hay diferencias por género, edad o nacionalidad.

Por otra parte, se establece que el entrenamiento en biorretroalimentación para controlar la respiración o la tasa cardiaca provoca que los sujetos puedan aplicar lo aprendido en sesión en contextos cotidianos con más facilidad que otros entrenamientos, propiciando que puedan controlar su respiración y tasa cardíaca de manera voluntaria al notar que comienzan a alterarse sus patrones fisiológicos, lo que potencialmente se cree permite detener una crisis asmática o al menos iniciar su control de una manera temprana, lo que reduce la severidad de la misma. En estos casos no se limita la intervención al trabajo en sesión, sino que se refuerza el trabajo con tareas para el hogar. Esto sucede, por ejemplo, en el caso de Kern-Buell et al. (2000), quienes recomendaron a las personas participantes seguir practicando las técnicas de relajación en sus casas, para lo cual les facilitaron las instrucciones grabadas, mientras que Georga et al. (2018) directamente entrenaron a los sujetos de su estudio para llevar a cabo las sesiones de biorretroalimentación en sus casas durante 8 semanas.

Adicionalmente, autores como Coen et al. (1996) sostienen que la relajación asistida con biorretroalimentación es beneficiosa para tratar la ansiedad y que esto tiene resultados positivos en los pacientes con asma, pues es ansiedad lo que experimentan al tener un episodio. Esto es similar a lo encontrado por Georga et al. (2018), quienes establecen que la relajación asistida con biorretroalimentación para el manejo del estrés mejora la calidad de vida de las personas con asma.

En cuanto al número de sesiones, Davis et al. (1973) y Khan (1977) reportan mejoras a partir de un número específico de sesiones. Los primeros reportan que el grupo de participantes con asma no severo mostró mejoras a partir de la tercera sesión con biorretroalimentación, mientras que el grupo control que solo recibía entrenamiento en relajación muscular progresiva mostró una leve tendencia a la mejora a partir de la quinta sesión. El segundo menciona que 27 niños del grupo experimental lograron reducir la 
resistencia respiratoria a partir de la quinta sesión, lo que llevó a la reducción de la frecuencia de los episodios.

A su vez Khan (1977) evaluó los cambios en la frecuencia y duración de los ataques y el número de visitas a la sala de emergencias de los participantes a lo largo del estudio. Debido a que el número de visitas a la sala de emergencias fue muy pequeño, en este aspecto no se encuentran diferencias significativas entre los grupos. Sin embargo, sí se reporta una mejoría significativa, donde el tratamiento con biorretroalimentación contribuye a la reducción de la frecuencia y duración de los episodios; y a una disminución en la severidad del asma y en el uso de medicamentos. No obstante, en los casos de asma de moderado a severo sí resulta necesario acompañar la intervención con el uso de medicación con esteroides.

Este último hallazgo ha sido replicado en estudios más recientes como los de Coen et al (1996), Kern-Buell et al. (2000) y O’Dwyer et al. (2020). No obstante, a partir de esta revisión sistemática es posible observar que en algunos estudios no se controla de manera rigurosa el uso de medicación por parte de los sujetos, por lo que el peso que tiene la medicación sobre estos resultados no es del todo claro y queda como interrogante de investigación. Asimismo, se considera que, ante la proporción de personas que sufren de asma en todo el mundo, el que puedan contar con estrategias que ayuden a mitigar los síntomas principales ante un ataque mejoraría su calidad de vida, aun cuando esto no sustituya su dependencia a medicamentos.

Por último, llama la atención que no se encontraron estudios nacionales que cumplieran con los criterios de validez aquí planteados, además de que no se encontraron muchos estudios en la zona latinoamericana que utilizaran este tipo de tecnología. Esto puede significar que en la región no se cuenta con ella o que no hay un verdadero interés por investigar estos tratamientos distintos al uso de medicación.

Sobre este último punto, si bien ha mejorado la tecnología con la que se fabrican los inhaladores y otros medicamentos para el asma, se considera que dichos avances no son suficientes para dejar de investigar sobre tratamientos alternativos y complementarios para este padecimiento. Hay muchas variables que pueden influir en el acceso a los medicamentos, desde el costo económico que estos implican, hasta casos de personas que 
presentan reacciones alérgicas, de forma que al no contarse con alternativas a este tratamiento farmacológico se afecta su calidad y condición de vida. En este sentido, la psicología basada en evidencia y especialmente el uso de biorretroalimentación dentro de la misma, implica el desarrollo de un abordaje que buscar mejorar la calidad de vida de las personas, mostrando una alternativa eficaz de tratamiento para algunos de sus síntomas. Dicho lo anterior, se visualiza la necesidad de seguir investigando en el tema y mantener actualizadas las opciones que personas con padecimientos crónicos como este, tienen para suplir sus afecciones.

\section{Conclusiones}

Si bien el uso de la biorretroalimentación para el tratamiento del asma no es un tema nuevo, se puede observar que conforme se avanzó en el desarrollo de fármacos se fueron dejando de lado los tratamientos alternativos y complementarios. En este contexto, los estudios realizados a mediados del siglo pasado contenían varias terapias alternativas a la medicación con el propósito de brindar opciones a las personas con esta condición; sin embargo, la cantidad de investigaciones con esta metodología fue reduciéndose en forma progresiva.

En la actualidad, el tratamiento con biorretroalimentación para el asma está categorizado como un nivel dos de eficacia (Frank et al. 2010). Esto implica que está respaldado por al menos un estudio con suficiente poder estadístico, con medidas bien identificadas, pero que carece de asignación aleatoria a condiciones de control a lo interno del estudio. En este escenario es importante realizar estudios que tomen en cuentas aspectos como la aleatorización, el uso de grupos control de listas de espera o tratamiento habitual, el registro de la muerte muestral y el control preciso del uso de medicación en los participantes, para poder responder de una manera más clara a la interrogante ¿Es la biorretroalimentación una alternativa para el tratamiento del asma respaldado por la evidencia? 
A partir de esta revisión sistemática se logra constatar que la biorretroalimentación en casos de asma leve o moderada, cuando es acompañada de otras técnicas, como la relajación y ejercicios de respiración, muestra excelentes resultados. Por ejemplo, contribuye a reducir la frecuencia y duración de las crisis asmáticas, el uso de medicación y la sintomatología en general (Davis 1973, Kahn 1977, Coen et al. 1996, Lehrer 2006, Kern-Buell et al. 2000, O'Dwyer et al. 2020). No obstante, resultados mixtos reportados en los estudios revisados, en conjunto con los alcances limitados que reportaron algunos estudios, hacen necesario clarificar que no es posible plantear la sustitución del tratamiento farmacológico en la totalidad de los casos, sino más bien en algunos casos particulares en donde la sintomatología es leve a moderada.

Por otra parte, la falta de estudios en Costa Rica y la zona de latinoamérica, a pesar de contar con gran cantidad de población que padece asma, puede relacionarse con la ignorancia y falta de interés sobre tratamientos además de los farmacológicos. La prevalencia de asma en Costa Rica en el 2017 fue de 5.79\% (Roser y Ritchie 2016), por lo que sería idóneo desarrollar estudios sobre este tema, tomando en cuenta criterios de validez científica, para generar información sobre los efectos de la biorretroalimentación aquí en la región. En este sentido, es importante equipar laboratorios con equipos de biorretroalimentación y adiestrar a profesionales para que se utilicen de rutina en las intervenciones.

Estas observaciones se plantean con la intención de llegar a una respuesta más clara sobre la eficacia de este tratamiento para el asma, y, en caso de que se considere eficaz, que cuente con el respaldo empírico que permita generar protocolos de intervención que se puedan utilizar en contextos clínicos y académicos. En este sentido, si bien es cierto que el equipo de biorretroalimentación es costoso, requiere de entrenamiento para usarse y hasta el apoyo de otra persona para manipularlo adecuadamente; con el tiempo ha surgido tecnología que permite tomar ese tipo de datos fisiológicos en un formato móvil y de forma más intuitiva para ser utilizado por particulares. En este escenario

[...] hay 2.32 billones de personas en el mundo que usan teléfonos inteligentes (smartphones). De esta forma, por ejemplo, los desarrollos de 
biosensores o de evaluación ecológica momentánea (EMA por sus siglas en inglés), también conocidos como métodos de muestreo experiencial, diarios electrónicos o evaluación ambulatoria, pueden permitir la recopilación de una gran cantidad de datos con mayor representatividad de lo que le ocurre a las personas en su vida real y que a su vez pueden analizarse mediante nuevos métodos estadísticos (Fernandez 2018).

Este tipo de abordajes podría contribuir a la personalización de los tratamientos y, por ende, significar una mejora considerable para la vida y salud de muchas personas. Sin embargo, aún falta comprobar la utilidad de estos dispositivos y la fiabilidad de los datos que puedan brindar.

\section{Referencias}

Coen, Barbara, Philip B, Conran, Angele McGrady y Lois Nelson.«Effects of biofeedback-Assisted Relaxation on Asthma Severity and Immune Function». Pediatric asthma, allergy \& immunology 10 (1996): 71-78. http://doi.org/10.1089/pai.1996.10.71

Davis, Margaret H., David R.Saunders, Thomas L.Creer y Hyman Chai. «Relaxation training facilitated by biofeedback apparatus as a supplemental treatment in bronchial asthma». Journal of Psychosomatic Research 17 (1973): 121-128. https://doi.org/10.1016/0022-3999(73)90012-3

Frank, Dana L., Lamees Khorshid, Jerome F. Kiffer, Christine S. Moravec y Michael G. Mckee. «biofeedback in medicine: who, when, why and how?». Mental Health in Family Medicine, 7 (2010): 85-91. PMCID: PMC2939454

Fernandez Alvarez, J.«Las tecnologías que transformarán la psicología clínica». Acceso el 3 de Setiembre de 2020, https://www.psyciencia.com/las-nuevas-tecnologiasque-transformaran-la-psicologia/

Georga, Georgia, George Chrousos, Artemios Artemiadis, Pelekasis P. Panagionista, Petros, Bakakosc y Christina, Darviri. «The effect of stress management incorporating progressive muscle relaxation and biofeedback-assisted relaxation breathing on patients with asthma: a randomised controlled trial». Advances in $\begin{array}{llll}\text { Integrative } & \text { Medicine, } & 6 & \text { 73-77. }\end{array}$ https://doi.org/10.1016/j.aimed.2018.09.001

Jiménez, Jennifer, «Costa Rica se coloca como uno de los países con la mayor cantidad de casos de asma infantil», Universidad de Costa Rica: Noticias Universidad de Costa Rica, 7 de marzo de 2019, acceso el 26 de agosto de 2020, 
Wimblu, Rev. Estud. de Psicología UCR, 16(1) 2021 (Enero-Junio): 29-50 /ISSN: 1659-2107

https://www.ucr.ac.cr/noticias/2019/03/07/costa-rica-se-coloca-como-uno-delos-paises-con-la-mayor-cantidad-de-casos-de-asmainfantil.html\#: :text=Dentro\%20de\%20estas\%20estad\%C3\%ADsticas\%2C\%20 Costa,a\%C3\%B1os\%20que\%20portan\%20el\%20padecimiento

Kern-Buell, Cheryl L., Angele V. McGrady, Philip B. Conran, and Lois A. Nelson. 2000. Asthma Severity, Psychophysiological Indicators Of Arousal, and Immune Function in Asthma Patients Undergoing Biofeedback-Assisted Relaxation. Applied Psychophysiology And Biofeedback $25 \quad$ (2): 79-91. doi:10.1023/a:1009562708112.

Khan, Aman U. «Effectiveness of biofeedback and Counter Conditioning In The Treatment of Bronchial Asthma». Journal of Psychosomatic Research, 21 (1977): 97-104. https://doi.org/10.1016/0022-3999(77)90078-2

Kondo, karli, Katherine M. Noonan, Michele Freeman, Chelsea Ayers, Benjamin J. Morasco, Devan Kansagara. «Efficacy of biofeedback for Medical Conditions: an Evidence Map». Journal Of General Internal Medicine, 34 (2019): 2883-2893. doi: 10.1007/s11606-019-05215-z

Luna, Alexandre J.H. de O., Philippe Kruchten, Marcello L. G. do E. Pedrosa, Humberto R. de AlmeidaNeto y Hermano P. de Moura. «State of the art of agile governance: a systematic review». International Journal of Computer Science \& Information Technology (IJCSIT), 6 (2014): 121-141. DOI:10.5121/ijcsit.2014.6510

Lehrer, Paul M., Stuart M. Hochron, Barbara McCann, Leora Swartzman, Phyllis Reba. «Relaxation decreases large-airway but not smallairway asthma». Journal of Psychosomatic Research, 30 (1986):13-25. https://doi.org/10.1016/0022$\underline{3999(86) 90061-9}$

Lehrer, Paul M., R. E. Carr, A. Smetankine, E. Vaschillo, E. Peper, S. Porges, R. Edelberg, R. Hamer, S. Hochron. «Respiratory Sinus Arrhythmia Versus Neck Trapezius EMG and Incentive Inspirometry biofeedback for Asthma: A Pilot Study». Applied PsychoPhysiology and biofeedback, 22(1997): 95-109

Lehrer, Paul M., Evgeny G. Vaschillo, Bronya Vaschillo, Shou-En Lu, Anthony T. Scardella, Mahmood Siddique, Robert H. Habib. «biofeedback Treatment for Asthma*». Chest Journal, 126(2004): 352-361. DOI:10.1378/chest.126.2.352

Lehrer, Paul M., Evgeny Vaschillo, Shou-En Lu, Dwain Eckberg, Bronya Vaschillo, Anthony Scardella y Robert Habib. «Heart Rate Variability biofeedback. Effects of Age on HRV, Baroreflex Gain and Asthma». CHEST, 129 (2006): 278-284. doi:10.1378/chest.129.2.278

Lehrer, Paul M. 2012. «Biofeedback Therapy for Asthma». En Functional Respiratory Disorders, editado por Ran D. Anbar, 179-205. New York: Springer.

Lehrer, Paul M., Charles G. Irvin, Shou-En Lu, Anthony Scardella, Beatrix RoehmheldHamm, Milisyaris Aviles-Velez, Jessica Graves, Evgeny G. Vaschillo, Bronya 
Vaschillo, Flavia Hoyte, Harold Nelson \& Frederick S. Wamboldt. «Heart Rate Variability biofeedback Does Not Substitute for Asthma Steroid Controller Medication». Appl Psychophysiol biofeedback, 43 (2017): 57-73. DOI 10.1007/s10484-017-9382-0

Long, K., Linda J. Ewing, Sheldon Cohen, David Skoner, Deborah Gentile, Jennifer Koehrsen, Chelsea Howe, Amanda L. Thompson, Rhonda K. Rosen, Megan Ganley, Anna L. Marsland. «Preliminary evidence for the feasibility of stress managment intervention for 7 to 12 year olds with asthma». Journal of asthma, 48 (2011): 162-170. DOI: 10.3109/02770903.2011.554941

Mussell, M. J. y J. Hartley. «Trachea-noise biofeedback in asthma: A comparison of the effect of trachea-noise biofeedback, a bronchodilator, and no treatment on the rate of recovery from Exercise- and eucapnic hyperventilation-induced asthma». biofeedback and Self-Regulation, 13 (1988): 219-234. DOI: https://doi.org/10.1007/BF00999171

Nelson, Krista L., Shou-En Lu, Tanya Oken, Paul M. Lehrer y Jonathan M. Feldman. «Further Exploration of Treatment Response in Latinos with Comorbid Asthma and Panic Disorder: A Brief Report of HRV and ETCO2 as Potential Mediators of Treatment Response». Applied Psychophysiology And biofeedback, 45(2020), 67-74. doi: 10.1007/s10484-020-09454-3

O'Dwyer, Susan, Garret Greene, Elaine MacHale, Breda Cushen, Imran Sulaiman, Fiona Boland, Sinthia Bosnic-Anticevich, Matshediso C. Mokoka, Richard B. Reilly, Terence Taylor, Sheila A. Rayder y Richard W. Costello. «Personalized Biofeedback on Inhaler Adherence and Technique by Community Pharmacists: A Cluster Randomized Clinical Trial». The Journal of Allergy and Clinical Immunology: In Practice, 8 (2020), 635-644. https://doi.org/10.1016/j.jaip.2019.09.008

Orozco Guzmán, M., \& Caballero Vallejo, K. Psicología Latinoamericana: experiencias, desafíos y compromisos sociales. Mexico: ALFEPSI, 2014. https://guardianes.org.mx/wp-content/themes/guardianes/pdf/psicologialatinoamericana.pdf

Ritz, Thomas, Bernard D. y Walton T. «Behavioral interventions in asthma». Journal of Psychosomatic Research, 56 (2004): 711-720. doi:10.1016/s00223999(03)00131-4

Roser, Max y Hannah Ritchie (2016). Burden of disease. Acceso el 26 de agosto de 2020, https://ourworldindata.org/burden-of-disease

Steptoe, Andrew, Jonathan Phillips y Jill Harling. «Biofeedback and instructions in the modification of total respiratory resistance: An experimental study of asthmatic and non-asthmatic volunteers». Journal of Psychosomatic Research, 25(1981): 541-551. DOI: https://doi.org/10.1016/0022-3999(81)90108-2 
Wimblu, Rev. Estud. de Psicología UCR, 16(1) 2021 (Enero-Junio): 29-50 /ISSN: 1659-2107

Taghizadeh, Niloofar, Aliereza Eslaminejad y Mohammad Reza Raoufy. «Protective effect of heart rate variability biofeedback on stress-induced lung function impairment in asthma». Respiratory Physiology \& Neurobiology (2019):49-56. DOI: $10.1016 /$ j.resp.2019.01.011

The Global Asthma Network. The Global Asthma Report 2018. Auckland:The Global Asthma Network, 2018. 